\title{
Control Design for Linear Strictly Metzler Time-delay Systems
}

\author{
DUŠAN KROKAVEC, ANNA FILASOVÁ \\ Department of Cybernetics and Artificial Intelligence \\ Faculty of Electrical Engineering and Informatics \\ Technical University of Košice \\ Letná 9, 04200 Košice \\ SLOVAKIA
}

\begin{abstract}
The relationships among structural constraints and involvement of the design condition are studied to synthesize state control for one class of linear strictly Metzler time-delay systems. These characterizations reflect the specific dynamical and structural attributes of the system class and outline the associated structures of linear matrix inequalities. Adjusting diagonal forms of linear matrix variables it is indicated how the proposed method gives a computable technique for the Metzler time-delay system, guaranteeing stabilising effect through implicit diagonal stabilization. The aim of this research is to describe conditions tying together inequality formulations and concepts of control theory in structures of Metzler systems.
\end{abstract}

Key-Words: - Time-delay Metzler systems, positive linear systems, diagonal stabilization, asymptotic stability, linear matrix inequalities.

Received: December 2, 2020. Revised: September 5, 2021. Accepted: September 13, 2021. Published: September 29, 2021.

\section{Introduction}

Positive systems allow obtaining representations of interesting properties of plants and their implementations. Among the main features can be mentioned the facts that stability of delayed positive systems does not depend on the amplitude of delays [1] and the pronouncement that in practice asymptotical stability of positive systems is equivalent to diagonal quadratical stability principle ([2], [3]). Using of appropriate algorithms it means analysis, stability and performance characterizations with positivity [4]. Moreover, the system positivity can be interpreted through the model parametric constraints to get more preferable design [5]. The used notation Metzler systems means that the research concepts prioritize Metzler matrix presentation [6]. Similar trends one can see in methods reflecting time delays in networked control systems ([7], [8], [9]) and in control design of Metzler time delayed systems, applying modern/advanced control techniques to design the feedback according to reasonable criteria ([10], [11], [12], [13]).

The main interest of the paper is to implement the state control for strictly Metzler linear time-delay systems (LTDS), when covering by linear matrix inequality (LMI) representation the system structural constraints. Facilitating the control structure implementation, the principle of diagonal stabilization provides a way to solve positive linear system synthesis using linear programming method ([14], [15]). To indicate that the resulting stable closed-loop system matrices are in general non-negative ([16], [17]) the preference for LMI-based forms is prioritized in [18].
Towards to the above and [19], whilst preferring new formulation, an LMI synthesis is established to guarantee strictly Metzler closed-loop system matrices if the time-delay system matrices are strictly Metzler. Although such defined multi-input, multi-output (MIMO) Metzler time-delay systems prescribe the set of parametric constraints, the proposed design conditions allow numerical solution in straightforward access, without combination of linear programming. The focus of this paper is an extension of methodology to construct systematically the control synthesis for a class of Metzler time-delay systems that go beyond those published in ([20], [21]).

The remaining of the article is lay out as follows: Sect. 2 deals with the preliminaries related to linear strictly Metzler continuous-time systems, whilst the relevant synthesis formulation is given in Sect. 3. The preferred mechanisms of adaptation in control design for time-delayed Metzler systems is addressed in Sect. 4. The performance of the proposed technique is illustrated by carrying out a detailed example in Sect. 5, also supporting related outlines of new points of view and conclusions, drafted in Sect. 6.

For sake of convenience $\boldsymbol{x}^{\mathrm{T}}, \boldsymbol{X}^{\mathrm{T}}$ denotes the transpose of the vector $\boldsymbol{x}$ and the matrix $\boldsymbol{X}$, respectively, $\boldsymbol{X} \prec 0(\preceq 0)$ reflects that a square matrix $\boldsymbol{X}$ is symmetric negative (semi)definite, $\boldsymbol{I}_{n}$ fits the $n$-th order unit matrix, diag $[\cdot]$ enters up the elements of a (block) diagonal matrix, $\rho(\boldsymbol{X})$ indicates eigenvalue spectrum of the square matrix $\boldsymbol{X}, \mathbb{R}$ stands for real numbers, $\mathbb{R}_{+}^{n \times r}$ is the set of non-negative matrices and $\mathbb{M}_{-+}^{n \times n}$ $\left(\mathrm{IM}_{-+\circ}^{n \times n}\right)$ is set of strictly (purely) Metzler matrices. 


\section{Basic Preliminaries}

It is considered the control design problem corresponding to a class of Meztler LTDS, which may be seen in the form

$$
\begin{gathered}
\dot{\boldsymbol{q}}(t)=\boldsymbol{A} \boldsymbol{q}(t)+\sum_{i=1}^{p} \boldsymbol{A}_{i} \boldsymbol{q}\left(t-\tau_{i}\right)+\boldsymbol{B} \boldsymbol{u}(t) \\
\boldsymbol{y}(t)=\boldsymbol{C} \boldsymbol{q}(t)
\end{gathered}
$$

where the constant time-delays satisfy the condition $0<\tau_{1}<\cdots<\tau_{p} \equiv \tau$ and the input $\boldsymbol{u}(t) \in \mathbb{R}^{r}$. Trajectories $\boldsymbol{q}(t) \in \mathbb{R}_{+}^{n}$ and outputs $\boldsymbol{y}(t) \in \mathbb{R}_{+}^{m}$ are nonnegative, $\boldsymbol{C} \in \mathbb{R}_{+}^{m \times n}, \boldsymbol{B} \in \mathbb{R}_{+}^{n \times r}$ are nonnegative matrices and $\left\{\boldsymbol{A}, \boldsymbol{A}_{1}, \cdots, \boldsymbol{A}_{p} \in \mathbb{M}_{-+}^{n \times n}\right\}$ are Metzler.

To show that the feedback law solves the regulator problem stated in the introduction the following statements, borrowed from the properties of positive systems, are presented.

Definition 1 [6] A matrix $\boldsymbol{A} \in \mathbb{M}_{-+o}^{n \times n}$ is purely Metzler if its diagonal elements are negative and its offdiagonal elements are nonnegative. A matrix $\boldsymbol{A} \in$ $\mathrm{M}_{-+}^{n \times n}$ is strictly Metzler if its diagonal elements are negative and its off-diagonal elements are positive. A Metzler matrix is stable if it is Hurwitz.

Definition 2 (adapted from [14]) Given any nonnegative initial condition $\phi(t) \in \mathbb{R}_{+}^{n}$ such that $\boldsymbol{q}(t)=$ $\phi(t)$ for $-\tau \leq t \leq 0$, the externally unforced system (1) is said to be positive if the corresponding trajectory is nonnegative $\left(\boldsymbol{q}(t) \in \mathbb{R}_{+}^{n}\right.$ for all $\left.t \geq 0\right)$.

Lemma 1 [14] Assuming that an externally unforced system (1) is asymptotically stable, $\boldsymbol{A} \in \mathrm{M}_{-+}^{n \times n}$ is Metzler and the matrices $\boldsymbol{A}_{1}, \cdots, \boldsymbol{A}_{p} \in \mathbb{R}_{+}^{n \times n}$ are nonnegative $\left(\boldsymbol{A}_{i} \geq 0\right.$ element wise for $i \in\langle 1, p\rangle$ ) then the composed matrix

$$
\boldsymbol{A}^{\diamond}=\boldsymbol{A}+\sum_{i=1}^{p} \boldsymbol{A}_{i}
$$

is strictly Metzler and Hurwitz.

Remark 1 Obviously, if considering that all matrices $\left\{\boldsymbol{A}, \boldsymbol{A}_{1}, \cdots, \boldsymbol{A}_{p} \in \mathbb{M}_{-+}^{n \times n}\right\}$ are strictly Metzler their sum must have the form of a strictly Metzler matrix. In the sense of (3), however in dependence on positions of zero elements in the matrices from this set, there can be a strictly Metzler matrix $\boldsymbol{A}^{\diamond}$ even for cases where all matrices are only purely Metzler, or one or more matrices in $\left\{\boldsymbol{A}, \boldsymbol{A}_{1}, \cdots, \boldsymbol{A}_{p} \in \mathbb{M}_{-+}^{n \times n}\right\}$ are strictly Metzler and the others are purely Metzler matrices, respectively even if at least one of the matrices is purely Metzler and the remaining matrices do not have the structure of Metzler matrices.
This means different interpretations of the tasks of analysis and synthesis of such systems and leads to solutions with different parametric boundaries on Metzler structures ([22], [23]).

Unless otherwise specified in the context, the matrix elements in the set

$$
\mathcal{A}=\left\{\boldsymbol{A}, \boldsymbol{A}_{1}, \cdots, \boldsymbol{A}_{p}\right\} \in \mathbb{M}_{-+}^{n \times n}
$$

are considered to be strictly Metzler matrices and the initial condition $\phi(t) \in \mathbb{R}_{++}^{n}$ is strictly positive.

Definition 3 [24] A matrix $\boldsymbol{L} \in \mathbb{R}^{n \times n}$ is a permutation matrix if exactly one item in each column and in each row is equal to 1 and all other elements are equal to 0 .

Remark 2 Keeping into account Definition 3 and considering a diagonal matrix $\boldsymbol{Y} \in \mathbb{R}^{n \times n}$ and the circulant permutation matrix $\boldsymbol{L}^{\mathrm{T}} \in \mathbb{R}^{n \times n}$ such defined that

$$
\boldsymbol{Y}=\operatorname{diag}\left[\begin{array}{llll}
y_{1} & y_{2} & \cdots & y_{n}
\end{array}\right], \quad \boldsymbol{L}^{\mathrm{T}}=\left[\begin{array}{cc}
\mathbf{0} & \boldsymbol{I}_{n-1} \\
1 & \mathbf{0}^{\mathrm{T}}
\end{array}\right]
$$

then

$$
\boldsymbol{L}^{\mathrm{T}} \boldsymbol{Y} \boldsymbol{L}=\operatorname{diag}\left[\begin{array}{llll}
y_{2} & \cdots & y_{n} & y_{1}
\end{array}\right]
$$

The next remark follows the realisation ideas presented in [25].

Remark 3 The control design task for positive systems, as well as for Metzler time-delay systems, requires to apply diagonal stabilization principle [2].

This principle can be visualized if a strictly Metzler $\boldsymbol{A} \in \mathbb{R}_{-+}^{n \times n}$. is represented with a rhombic form, constructed by using sequentially circular shifts of growing order to the rows of $\boldsymbol{A}$ as follows [25]

$$
\begin{aligned}
& \boldsymbol{A}_{\Theta}=
\end{aligned}
$$

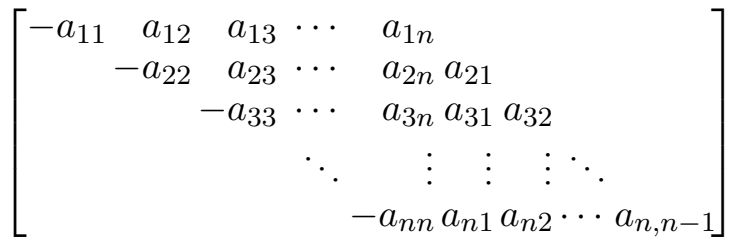

where the diagonal matrices for $h=1, \ldots, n-1$

$$
\begin{aligned}
& \quad \boldsymbol{A}_{\Theta}(l, l)=\operatorname{diag}\left[-a_{11}-a_{22} \cdots-a_{n n}\right] \\
& \boldsymbol{A}_{\Theta}(l, l+h)= \\
& \operatorname{diag}\left[a_{1,1+h} \cdots a_{n-h, n} a_{n-h+1,1} \cdots a_{n, h}\right]
\end{aligned}
$$

are related to the associated diagonals of $\boldsymbol{A}_{\Theta}$ and $\Theta=(1 \leftrightarrow n) / n$ notes the sum modulo $n+1$.

Moreover, it can see that generally $n^{2}$ Metzler matrix parametric constraints on $a_{i j}$ for $i, j \in\langle 1, n\rangle$ can be defined by the negativeness of (8) and positiveness of the set of diagonal matrices given in (9). 
Lemma 1 [18] Let the matrix $\boldsymbol{A} \in \mathbb{M}_{-+}^{n \times n}$ is strictly Metzler then it is Hurwitz if and only if there exists diagonal positive definite matrix (PDM) $\boldsymbol{S} \in \mathbb{R}_{+}^{n \times n}$ such that for $h=1, \ldots, n-1$ and circulant $\boldsymbol{L} \in \mathbb{R}_{+}^{n \times n}$ the following sets of LMIs is feasible

$$
\begin{gathered}
\boldsymbol{S} \succ 0 \\
\boldsymbol{A} \boldsymbol{S}+\boldsymbol{S} \boldsymbol{A}^{\mathrm{T}} \prec 0 \\
\boldsymbol{A}_{\Theta}(l, l) \boldsymbol{S} \prec 0 \\
\boldsymbol{L}^{h} \boldsymbol{A}_{\Theta}(l, l+h) \boldsymbol{L}^{h \mathrm{~T}} \boldsymbol{S} \succ 0
\end{gathered}
$$

If the task is connected with the control law

$$
\boldsymbol{u}(t)=-\boldsymbol{K} \boldsymbol{q}(t)
$$

it is not certain beforehand that it can in general find a strictly Metzler closed-loop system matrix. The following lemma gives a clue to the solution of this problem.

Lemma 2 [26] If a strictly Metzler $\boldsymbol{A} \in \mathbb{M}_{-+}^{n \times n}, a$ nonnegative $\boldsymbol{B} \in \mathbb{R}_{+}^{n \times r}$ and a positive $\boldsymbol{K} \in \mathbb{R}_{++}^{r \times n}$ guarantee that $\boldsymbol{A}_{c}=\boldsymbol{A}-\boldsymbol{B} \boldsymbol{K} \in \mathbb{M}_{-+}^{n \times n}$ is strictly Metzler then it yields for defined $h$ and $j$

$$
\boldsymbol{A}_{c}=\sum_{h=0}^{n-1}\left(\boldsymbol{A}_{\Theta}(l, l+h)-\sum_{j=0}^{r} \boldsymbol{B}_{j} \boldsymbol{K}_{d j h}\right) \boldsymbol{L}^{h \mathrm{~T}}
$$

where with $\boldsymbol{K}_{d j}, \boldsymbol{B}_{d j} \in \mathbb{R}_{+}^{n \times n}$

$$
\begin{aligned}
& \boldsymbol{A}_{\Theta}(l, l)-\sum_{j=0}^{r} \boldsymbol{B}_{j} \boldsymbol{K}_{d j} \prec 0 \\
& \boldsymbol{A}_{\Theta}(l, l+h)-\sum_{j=0}^{r} \boldsymbol{B}_{j} \boldsymbol{K}_{d j h} \succ 0 \\
& \boldsymbol{K}=\left[\begin{array}{c}
\boldsymbol{k}_{1}^{\mathrm{T}} \\
\vdots \\
\boldsymbol{k}_{r}^{\mathrm{T}}
\end{array}\right], \quad \boldsymbol{B}=\left[\boldsymbol{b}_{1} \cdots \boldsymbol{b}_{r}\right] \\
& \boldsymbol{K}_{d j}=\operatorname{diag}\left[\boldsymbol{k}_{j}^{\mathrm{T}}\right]=\operatorname{diag}\left[k_{j 1} \cdots k_{j n}\right] \\
& \boldsymbol{B}_{d j}=\operatorname{diag}\left[\boldsymbol{b}_{j}\right]=\operatorname{diag}\left[b_{j 1} \quad \cdots \quad b_{j n}\right] \\
& \boldsymbol{K}_{d j h}=\boldsymbol{L}^{h \mathrm{~T}} \boldsymbol{K}_{d j} \boldsymbol{L}^{h}
\end{aligned}
$$

Note, (16), (17) force parameter constraints of the Metzler $\boldsymbol{A}_{c}$ and do not guarantee that $\boldsymbol{A}_{c}$ is Hurwitz. Equation (15) together with (18)-(21) give the relations between the square and rhombic matrix representation of $\boldsymbol{A}_{c}$.

The control design task statement for time-delayfree Matzler systems is presented in the following section.

\section{Interpretation of Diagonal Stabilization Principle}

Basic facts in the design subject application for timedelay-free Matzler systems are summarized in the proof of this theorem.

Theorem 1 If for strictly Metzler $\boldsymbol{A} \in \mathbb{M}_{-+}^{n \times n}$ and nonnegative $\boldsymbol{B} \in \mathbb{R}_{+}^{n \times r}$ there exist diagonal PDMs $\boldsymbol{S}, \boldsymbol{R}_{j} \in \mathbb{R}_{+}^{n \times n}$ such that for $h=1, \ldots, n-1, j=$ $1, \ldots, r$ and the circulant $\boldsymbol{L} \in \mathbb{R}_{+}^{n \times n}$ is feasible the set of LMIs

$$
\boldsymbol{S} \succ 0, \quad \boldsymbol{R}_{j} \succ 0
$$

$$
\begin{aligned}
& \boldsymbol{A} \boldsymbol{S}+\boldsymbol{S} \boldsymbol{A}^{\mathrm{T}}- \\
- & \sum_{j=1}^{r} \boldsymbol{B}_{d j} \boldsymbol{l} \boldsymbol{l}^{\mathrm{T}} \boldsymbol{R}_{j}-\sum_{j=1}^{r} \boldsymbol{R}_{j} \boldsymbol{l l} \boldsymbol{l}^{\mathrm{T}} \boldsymbol{B}_{d j} \\
\prec & 0
\end{aligned}
$$

$$
\boldsymbol{A}_{\Theta}(l, l) \boldsymbol{L}^{h \mathrm{~T}} \boldsymbol{S}-\sum_{j=0}^{r} \boldsymbol{B}_{d j} \boldsymbol{R}_{j} \prec 0
$$

$$
\boldsymbol{L}^{h} \boldsymbol{A}_{\Theta}(l, l+h) \boldsymbol{L}^{h \mathrm{~T}} \boldsymbol{S}-\sum_{j=0}^{r} \boldsymbol{L}^{h} \boldsymbol{B}_{d j} \boldsymbol{L}^{h \mathrm{~T}} \boldsymbol{R}_{j} \succ 0
$$

where $\boldsymbol{l} \in \mathbb{R}_{+}^{n}$ is the $n$-dimensional vector of the form

$$
\boldsymbol{l}^{\mathrm{T}}=\left[\begin{array}{llll}
1 & 1 & \cdots & 1
\end{array}\right]
$$

then $\boldsymbol{K} \in \mathbb{R}_{++}^{r \times n}$ brings up in the form

$$
\boldsymbol{K}_{d j}=\boldsymbol{R}_{j} \boldsymbol{S}^{-1}, \boldsymbol{k}_{j}^{\mathrm{T}}=\boldsymbol{l}^{\mathrm{T}} \boldsymbol{K}_{d j}, \boldsymbol{K}=\left[\begin{array}{c}
\boldsymbol{k}_{1}^{\mathrm{T}} \\
\vdots \\
\boldsymbol{k}_{r}^{\mathrm{T}}
\end{array}\right]
$$

to apply stable control by constructing a strictly Metzler and Hurwitz $\boldsymbol{A}_{c}=\boldsymbol{A}-\boldsymbol{B} \boldsymbol{K} \in \mathbb{M}_{-+}^{n \times n}$.

Hereafter, $*$ denotes the symmetric item in a symmetric matrix.

Proof: The time-delay free system (1) subject to control given by (14) is stabilized with the choice support of Lyapunov function

$$
v(\boldsymbol{q}(t))=\boldsymbol{q}^{\mathrm{T}}(t) \boldsymbol{P} \boldsymbol{q}(t)>0
$$

if the time derivative of (28) is negative that is if

$$
\dot{v}(\boldsymbol{q}(t))=\dot{\boldsymbol{q}}^{\mathrm{T}}(t) \boldsymbol{P} \boldsymbol{q}(t)+\boldsymbol{q}^{\mathrm{T}}(t) \boldsymbol{P} \dot{\boldsymbol{q}}(t)<0
$$

where, with the valid diagonal stabilisation principle, $\boldsymbol{P} \in \mathbb{R}_{+}^{n \times n}$ is a diagonal PDM.

Letting

$$
\dot{\boldsymbol{q}}(t)=(\boldsymbol{A}-\boldsymbol{B} \boldsymbol{K}) \boldsymbol{q}(t)
$$

inequality (29) can be rewritten as

$$
\dot{v}(\boldsymbol{q}(t))=\boldsymbol{q}^{\mathrm{T}}(t)\left(\boldsymbol{A}_{c}^{\mathrm{T}} \boldsymbol{P}+\boldsymbol{P} \boldsymbol{A}_{c}\right) \boldsymbol{q}(t)<0
$$


where

$$
\boldsymbol{A}_{c}=\boldsymbol{A}-\boldsymbol{B K}
$$

Here $\boldsymbol{A}_{c}, \boldsymbol{K}$ is of the dimension $\boldsymbol{A}_{c} \in \mathbb{M}_{-+}^{r \times n}, \boldsymbol{K} \in$ $\mathbb{R}_{+}^{r \times n}$, respectively.

The negativeness of (29) is in correspondence with the negativeness of the matrix inequality

$$
\begin{aligned}
& \boldsymbol{P} \boldsymbol{A}_{c}+\boldsymbol{A}_{c}^{\mathrm{T}} \boldsymbol{P} \\
= & \boldsymbol{P}(\boldsymbol{A}-\boldsymbol{B} \boldsymbol{K})+(\boldsymbol{A}-\boldsymbol{B} \boldsymbol{K})^{\mathrm{T}} \boldsymbol{P} \\
\prec & 0
\end{aligned}
$$

Since the design method has to rely on linear matrix inequalities, introducing the diagonal positive definite matrix $\boldsymbol{S}=\boldsymbol{P}^{-1} \in \mathbb{R}_{+}^{r \times n}$ and pre-multiplying the left side and post-multiplying the right side by $\boldsymbol{S}$ then the bilinear (33) implies

$$
(\boldsymbol{A}-\boldsymbol{B} \boldsymbol{K}) \boldsymbol{S}+\boldsymbol{S}(\boldsymbol{A}-\boldsymbol{B} \boldsymbol{K})^{\mathrm{T}} \prec 0
$$

which is negative definite and linear.

Allowing the formalisation via diagonal matrix parameters (34) can be turned out to

$$
\boldsymbol{A} \boldsymbol{S}+\boldsymbol{S} \boldsymbol{A}^{\mathrm{T}}-\sum_{j=1}^{r} \boldsymbol{b}_{j} \boldsymbol{k}_{j}^{\mathrm{T}} \boldsymbol{S}-\sum_{j=1}^{r} \boldsymbol{S} \boldsymbol{k}_{j} \boldsymbol{b}_{j}^{\mathrm{T}} \prec 0
$$

which provides a means to apply (19), (20) as

$$
\begin{aligned}
& \boldsymbol{A} \boldsymbol{S}+\boldsymbol{S} \boldsymbol{A}^{\mathrm{T}}- \\
- & \sum_{j=1}^{r} \boldsymbol{B}_{d j} \boldsymbol{l} \boldsymbol{l}^{\mathrm{T}} \boldsymbol{K}_{d j} \boldsymbol{S}-\sum_{j=1}^{r} \boldsymbol{S} \boldsymbol{K}_{d j} \boldsymbol{l l} \boldsymbol{B}^{\mathrm{T}} \boldsymbol{B}_{d j} \\
\prec & 0
\end{aligned}
$$

where $l \in \mathbb{R}_{+}^{n}$ is the $n$-dimensional vector of the form (26). Thus, it can be readily shown that with

(36) implies (23).

$$
\boldsymbol{R}_{j}=\boldsymbol{S} \boldsymbol{K}_{d j}
$$

Multiplying the right side of (16) by $S$ it yields

$$
\boldsymbol{A}_{\Theta}(l, l) \boldsymbol{S}-\sum_{j=0}^{r} \boldsymbol{B}_{d j} \boldsymbol{K}_{d j} \boldsymbol{S} \prec 0
$$

and pre-multiplying the left side by $\boldsymbol{L}^{h}$ and post-multiplying the right side by $\boldsymbol{L}^{h \mathrm{~T}} \boldsymbol{S}$ then (17), 21) imply

$$
\boldsymbol{L}^{h} \boldsymbol{A}_{\Theta}(l, l+h) \boldsymbol{L}^{h \mathrm{~T}} \boldsymbol{S}-\sum_{j=0}^{r} \boldsymbol{L}^{h} \boldsymbol{B}_{d j} \boldsymbol{L}^{h \mathrm{~T}} \boldsymbol{K}_{d j} \boldsymbol{S} \succ 0
$$

Thus, substituting (37) then (38), (39) imply (24), (25). This completes the proof.

Remark 4 The condition (24) guarantees that the main diagonal elements of $\boldsymbol{A}_{c}$ are negative and LMIs (25) guarantee that the off-diagonal elements of $\boldsymbol{A}_{c}$ are strictly positive if $\boldsymbol{K} \in \mathbb{R}_{++}^{r \times n}$ and $\boldsymbol{B} \in \mathbb{R}_{+}^{n \times r}$. The Lyapunov matrix inequality (23) forces that $\boldsymbol{A}_{c}$ will be also Hurwitz.

\section{Control Design for Strictly Metzler Time-delay Systems}

The considered problem is connected with the control law (14) to guarantee that the equivalent to (3)

$$
\boldsymbol{A}_{c}^{\circ}=\boldsymbol{A}_{c}+\sum_{i=1}^{p} \boldsymbol{A}_{i}
$$

will be satisfied for given class of Metzler time-delay linear systems, where $\boldsymbol{A}_{c}^{\circ} \in \mathbb{M}_{-+}^{m \times n}$ for a positive control gain $\boldsymbol{K} \in \mathbb{R}_{+}^{r \times n}$ is strictly Metzler and Hurwitz.

Reflecting the diagonal stabilisation and letting

$$
\boldsymbol{A}_{s}=\sum_{i=1}^{p} \boldsymbol{A}_{i}
$$

then, if $\boldsymbol{A}_{s} \in \mathbb{M}_{-+}^{n \times n}$ is strictly Metzler, $\boldsymbol{A}_{c}^{\circ} \in \mathbb{M}_{-+}^{m \times n}$ has to be strictly Metzler when $\boldsymbol{A}_{c} \in \mathbb{M}_{-+}^{m \times n}$ is strictly Metzler and no additional parametric constraints need be added to the synthesis.

Thus, the principle presented in the previous section can by applied to the above specifications and this environment is paradigmatic to account for stable Metzler structures. The following theorem define the design task if $\boldsymbol{A}_{s} \in \mathbb{M}_{-+}^{n \times n}$ is Hurwitz.

Theorem 2 If for given strictly Metzler matrix $\boldsymbol{A} \in$ $\mathrm{M}_{-+}^{n \times n}$, strictly Metzler and Hurwitz matrix $\boldsymbol{A}_{s} \in$ $\mathbb{M}_{-+o}^{n \times n}$ and nonnegative matrix $\boldsymbol{B} \in \mathbb{R}_{+}^{n \times r}$ there exist diagonal PDMs $\boldsymbol{S}, \boldsymbol{Q}, \boldsymbol{R}_{j} \in \mathbb{R}_{+}^{n \times n}$ such that for $h=1,2, \ldots, n-1$ and $j=1,2, \ldots, r$, the circulant $\boldsymbol{L} \in \mathbb{R}_{+}^{n \times n}$ and $\boldsymbol{l} \in \mathbb{R}_{+}^{n}$ the following LMIs

$$
\boldsymbol{S} \succ 0, \quad \boldsymbol{Q} \succ 0, \quad \boldsymbol{R}_{j} \succ 0
$$

$$
\begin{aligned}
& \boldsymbol{A} \boldsymbol{S}+\boldsymbol{S} \boldsymbol{A}^{\mathrm{T}}+ \\
+ & \boldsymbol{A}_{s}(\boldsymbol{S}-\boldsymbol{Q})+(\boldsymbol{S}-\boldsymbol{Q}) \boldsymbol{A}_{s}^{\mathrm{T}}- \\
- & \sum_{j=1}^{r} \boldsymbol{B}_{d j} \boldsymbol{l} \boldsymbol{l}^{\mathrm{T}} \boldsymbol{R}_{j}-\sum_{j=1}^{r} \boldsymbol{R}_{j} \boldsymbol{l} \boldsymbol{l}^{\mathrm{T}} \boldsymbol{B}_{d j} \\
\prec & 0
\end{aligned}
$$

$$
\boldsymbol{A}_{s} \boldsymbol{Q}+\boldsymbol{Q} \boldsymbol{A}_{s}^{\mathrm{T}} \prec 0
$$

$$
\boldsymbol{A}_{\Theta}(l, l) \boldsymbol{L}^{h \mathrm{~T}} \boldsymbol{S}-\sum_{j=0}^{r} \boldsymbol{B}_{d j} \boldsymbol{R}_{j} \prec 0
$$

$$
\boldsymbol{L}^{h} \boldsymbol{A}_{\Theta}(l, l+h) \boldsymbol{L}^{h \mathrm{~T}} \boldsymbol{S}-\sum_{j=0}^{r} \boldsymbol{L}^{h} \boldsymbol{B}_{j} \boldsymbol{L}^{h \mathrm{~T}} \boldsymbol{R}_{j} \succ 0
$$

have a feasible performance solution, then the gain $\boldsymbol{K} \in \mathbb{R}_{++}^{r \times n}$ is given as in 27) and the matrices $\boldsymbol{A}_{c} \in$ $\mathbb{M}_{-+}^{n \times n}, \boldsymbol{A}_{c}^{\circ} \in \mathbb{M}_{-+}^{n \times n}$ are strictly Metzler and Hurwitz. 
Proof: The consistent specification and the control scheme (14) result that the stability condition (34) can be re-specified as

$$
\left(\boldsymbol{A}+\boldsymbol{A}_{s}-\boldsymbol{B} \boldsymbol{K}\right) \boldsymbol{S}+\boldsymbol{S}\left(\boldsymbol{A}+\boldsymbol{A}_{s}-\boldsymbol{B} \boldsymbol{K}\right)^{\mathrm{T}} \prec 0
$$

considering a diagonal PDM $\boldsymbol{P} \in \mathbb{R}_{+}^{n \times n}$, a strictly Metzler $\boldsymbol{A} \in \mathrm{IM}_{-+}^{n \times n}$ and a strictly Metzler and Hurwitz $\boldsymbol{A}_{s} \in \mathbb{M}_{-+\circ}^{n \times n}$.

Since $\boldsymbol{A}_{s}$ is strictly Metzler and Hurwitz in the sense of Lyapunov there exists a diagonal PDM $Q \in$ $\mathbb{R}_{+}^{n \times n}$ such that 24$)$ is satisfied.

These attributes specify the closed-loop system stability properties when $(36)$ is extendibly reformulated as follows

$$
\begin{aligned}
& \boldsymbol{A} \boldsymbol{S}+\boldsymbol{S} \boldsymbol{A}^{\mathrm{T}}+\boldsymbol{A}_{s} \boldsymbol{S}+\boldsymbol{S} \boldsymbol{A}_{s}^{\mathrm{T}}- \\
- & \sum_{j=1}^{r} \boldsymbol{b}_{j} \boldsymbol{k}_{j}^{\mathrm{T}} \boldsymbol{S}-\sum_{j=1}^{r} \boldsymbol{S} \boldsymbol{k}_{j} \boldsymbol{b}_{j}^{\mathrm{T}} \\
= & \boldsymbol{A} \boldsymbol{S}+\boldsymbol{S} \boldsymbol{A}^{\mathrm{T}}+\boldsymbol{A}_{s} \boldsymbol{S}+\boldsymbol{S} \boldsymbol{A}_{s}^{\mathrm{T}}- \\
- & \sum_{j=1}^{r} \boldsymbol{B}_{d j} \boldsymbol{l} \boldsymbol{l}^{\mathrm{T}} \boldsymbol{K}_{d j} \boldsymbol{S}-\sum_{j=1}^{r} \boldsymbol{S} \boldsymbol{K}_{d j} \boldsymbol{l} \boldsymbol{l}^{\mathrm{T}} \boldsymbol{B}_{d j} \\
\preceq & \boldsymbol{A}_{s} \boldsymbol{Q}+\boldsymbol{Q} \boldsymbol{A}_{s}^{\mathrm{T}} \\
\prec & 0
\end{aligned}
$$

Providing that the negative definite $\boldsymbol{A}_{s} \boldsymbol{Q}+\boldsymbol{Q} \boldsymbol{A}_{s}^{\mathrm{T}}$ is relocated in the left side and (37) is substituted then (48) implies (43).

Since there is considered that $\boldsymbol{A}_{c} \in \mathbb{M}_{-+}^{m \times n}$ has to be strictly Metzler, the parametric constraints can be done by directly specifying the same matrix inequalities (24), (25), which imply (44), (45). This completes the proof.

Remark 5 If LMIs (42)-(46) being feasible, the conditions (45)-(46) cover that the matrix $\boldsymbol{A}_{c}$ will be strictly Metzler, the matrix inequality (44) clamps that $\boldsymbol{A}_{s}$ is Metzler and Hurwitz, (43) defines that $\boldsymbol{A}_{c}, \boldsymbol{A}_{c}^{\circ}$ will be also Metzler and Hurwitz and the positive definite diagonal matrix variables $\boldsymbol{S}, \boldsymbol{R}_{j}$ guarantee that $\boldsymbol{K} \in \mathbb{R}_{+}^{r \times n}$ is positive. Since both closed-loop related system matrices $\boldsymbol{A}_{s}, \boldsymbol{A}_{c}$ are strictly Metzler then, evidently, $\boldsymbol{A}_{c}^{\circ}$ is strictly Metzler.

The perception of the control task for an unstable Metzler matrix $\boldsymbol{A}_{s}$ is formally equivalent to the above established design problem, but the Lyapunov matrix inequality (44) cannot be included in the LMIs defining the closed-loop stability conditions.

To find the control law parameter, which produces the internally stable closed-loop with acceptable performances for a strictly Metzler system with instability in the delay-free and delayed system matrices, can be proved using the above principles in the following theorem.
Corollary 1 If for given Hurwitz $\boldsymbol{A}_{s} \in \mathbb{M}_{-+\circ}^{n \times n}$, unstable strictly Metzler matrix $\boldsymbol{A} \in \mathbb{M}_{-+}^{n \times n}$, and nonnegative matrix $\boldsymbol{B} \in \mathbb{R}_{+}^{n \times r}$ there exist diagonal PDMs $\boldsymbol{S}, \boldsymbol{R}_{j} \in \mathbb{R}_{+}^{n \times n}$ such that for $h=1,2, \ldots, n-$ 1 and $j=1,2, \ldots, r$, the circulant $\boldsymbol{L} \in \mathbb{R}_{+}^{n \times n}$ and $\boldsymbol{l} \in \mathbb{R}_{+}^{n}$ the only feasible set of LMIs

$$
\boldsymbol{S} \succ 0, \quad \boldsymbol{R}_{j} \succ 0
$$

$$
\begin{aligned}
& \boldsymbol{A} \boldsymbol{S}+\boldsymbol{S} \boldsymbol{A}^{\mathrm{T}}+\boldsymbol{A}_{s} \boldsymbol{S}+\boldsymbol{S} \boldsymbol{A}_{s}^{\mathrm{T}}- \\
- & \sum_{j=1}^{r} \boldsymbol{B}_{d j} \boldsymbol{l} \boldsymbol{l}^{\mathrm{T}} \boldsymbol{R}_{j}-\sum_{j=1}^{r} \boldsymbol{R}_{j} \boldsymbol{l} \boldsymbol{l}^{\mathrm{T}} \boldsymbol{B}_{d j} \\
\prec & 0
\end{aligned}
$$

$$
\boldsymbol{A}_{\Theta}(l, l) \boldsymbol{L}^{h \mathrm{~T}} \boldsymbol{S}-\sum_{j=0}^{r} \boldsymbol{B}_{d j} \boldsymbol{R}_{j} \prec 0
$$

$$
\boldsymbol{L}^{h} \boldsymbol{A}_{\Theta}(l, l+h) \boldsymbol{L}^{h \mathrm{~T}} \boldsymbol{S}-\sum_{j=0}^{r} \boldsymbol{L}^{h} \boldsymbol{B}_{j} \boldsymbol{L}^{h \mathrm{~T}} \boldsymbol{R}_{j} \succ 0
$$

gives $\boldsymbol{K} \in \mathbb{R}_{++}^{r \times n}$ as in $(27)$, the strictly Metzler $\boldsymbol{A}_{c} \in$ $\mathbb{M}_{-+}^{n \times n}$ and strictly Metzler and Hurwitz $\boldsymbol{A}_{c}^{\circ} \in \mathbb{M}_{-+}^{n \times n}$, respectively.

Remark 6 Since in this case (48) reduces to

$$
\begin{aligned}
& \boldsymbol{A} \boldsymbol{S}+\boldsymbol{S} \boldsymbol{A}^{\mathrm{T}}+\boldsymbol{A}_{s} \boldsymbol{S}+\boldsymbol{S} \boldsymbol{A}_{s}^{\mathrm{T}}- \\
- & \sum_{j=1}^{r} \boldsymbol{B}_{d j} \boldsymbol{l l}^{\mathrm{T}} \boldsymbol{K}_{d j} \boldsymbol{S}-\sum_{j=1}^{r} \boldsymbol{S} \boldsymbol{K}_{d j} \boldsymbol{l l}^{\mathrm{T}} \boldsymbol{B}_{d j} \\
\prec & 0
\end{aligned}
$$

substituting (37) then (53) implies (50). Because in order to ensure strictly Metzler matrix structures, (51), (52) are still required.

Remark 7 If unstable strictly Metzler matrix $\boldsymbol{A} \in$ $\mathbb{M}_{-+}^{n \times n}$ and Hurwitz $\boldsymbol{A}_{s} \in \mathbb{M}_{-+\circ}^{n \times n}$ are normcomparable, $\boldsymbol{A}_{c}^{\circ} \in \mathbb{M}_{-+}^{n \times n}$ is strictly Metzler and Hurwitz and $\boldsymbol{K} \in \mathbb{R}_{+}^{r \times n}$ is positive, with a positive scalar $\alpha \in \mathbb{R}_{+}$can be formed hypothetically separated two matrices

$$
\begin{gathered}
\boldsymbol{A}_{c}^{\alpha}=\boldsymbol{A}-\alpha \boldsymbol{B} \boldsymbol{K} \\
\boldsymbol{A}_{c s}^{\alpha}=\boldsymbol{A}_{s}-(1-\alpha) \boldsymbol{B} \boldsymbol{K}
\end{gathered}
$$

to be strictly Metzler and Hurwitz. This establish limitations on achieved feedback control properties.

Remark 8 Consider that $\boldsymbol{B} \in \mathbb{R}_{+}^{n \times r}$ is nonnegative, $\boldsymbol{A} \in \mathbb{M}_{-+o}^{n \times n}$ is purely Metzler, $\boldsymbol{A}_{s} \in \mathbb{M}_{-+\circ}^{n \times n}$ is purely Metzler and Hurwitz whilst these matrices are offdiagonally compatible with respect to zero elements (a compatible zero element is on the row number $z$ and the column number $y$ in both $\boldsymbol{A}$ and $\boldsymbol{A}_{s}$ ). 
If the $z$-th row of $\boldsymbol{B}$ is zero vector and the offdiagonal zero element in both matrices $\boldsymbol{A}, \boldsymbol{A}_{s}$ is on the position with the row number $z$ the unlimited design conditions remain as given in Theorem 2 and in the feasible case the result is a positive $\boldsymbol{K} \in \mathbb{R}_{+}^{r \times n}$, implying purely Metzler matrices and Hurwitz matrices $\boldsymbol{A}_{c}, \boldsymbol{A}_{c}^{\circ} \in \mathbb{M}_{-+\circ}^{n \times n}$. In the opposite case when elements of the $z$-th rov of $\boldsymbol{B}$ are nonzero then the conditions defined by Theorem 2 must be reformulated for the structured positive semi-definite diagonal matrix variables $\boldsymbol{R}_{j}$ as

$$
\boldsymbol{R}_{j}=\operatorname{diag}\left[r_{j 1} \cdots r_{j, z-1} r_{j z} r_{j, z+1} \cdots r_{j n}\right] \succeq 0
$$

where for all $j$ and given $z$ is $r_{j z}=0$.

The results in the last mentioned case mean nonnegative $\boldsymbol{K} \in \mathbb{R}_{+}^{r \times n}$ and purely Metzler and Hurwitz matrices $\boldsymbol{A}_{c}, \boldsymbol{A}_{c}^{\circ} \in \mathbb{M}_{-+\circ}^{n \times n}$. The inclusion of the reference [27] makes up for the omitted details of the explanation. Other cases, if they are solvable, can be formulated "ad hoc" in definition of structural positive semi-definite diagonal matrix variables $\boldsymbol{R}_{j}$.

\section{Illustrative example}

The proposed design algorithm (42)-(46) starts from the model (1), (2), defined for $p=2$ by

$$
\begin{aligned}
& \boldsymbol{A}=\left[\begin{array}{rrr}
-0.086 & 1.032 & 1.290 \\
0.086 & -3.268 & 0.258 \\
0.344 & 0.602 & -2.408
\end{array}\right], \boldsymbol{B}=\left[\begin{array}{ll}
0.5 & 1.0 \\
1.0 & 0.9 \\
0.7 & 1.1
\end{array}\right] \\
& \boldsymbol{A}_{1}=\left[\begin{array}{rrr}
-0.108 & 0.776 & 0.580 \\
0.004 & -1.584 & 0.080 \\
0.180 & 0.280 & -1.164
\end{array}\right] \\
& \boldsymbol{A}_{2}=\left[\begin{array}{rrr}
-0.104 & 0.824 & 0.620 \\
0.006 & -1.456 & 0.144 \\
0.100 & 0.312 & -1.020
\end{array}\right] \\
& \boldsymbol{A}_{s}=\left[\begin{array}{rrr}
-0.212 & 1.600 & 1.200 \\
0.010 & -3.040 & 0.224 \\
0.280 & 0.592 & -2.184
\end{array}\right], \boldsymbol{C}^{\mathrm{T}}=\left[\begin{array}{ll}
1 & 0 \\
0 & 1 \\
0 & 0
\end{array}\right]
\end{aligned}
$$

where $\boldsymbol{A}_{1}, \boldsymbol{A}_{2}, \boldsymbol{A}_{s}$ are strictly Metzler and Hurwitz and the unstable $\boldsymbol{A}$ is strictly Metzler.

With synthesis focusing points, of immediate consequences are observed the structures related to the underlying system description

$$
\begin{aligned}
\boldsymbol{L}^{\mathrm{T}}=\left[\begin{array}{ll}
\mathbf{0} & \boldsymbol{I}_{2} \\
1 & \mathbf{0}^{\mathrm{T}}
\end{array}\right], \quad \boldsymbol{l}^{\mathrm{T}}=\left[\begin{array}{ll}
1 & 1
\end{array}\right] \\
\boldsymbol{A}_{\Theta}(l, l)=-\operatorname{diag}\left[\begin{array}{lll}
0.086 & 3.268 & 2.4080
\end{array}\right] \\
\boldsymbol{A}_{\Theta}(l, l+1)=\operatorname{diag}\left[\begin{array}{lll}
1.032 & 0.258 & 0.344
\end{array}\right] \\
\boldsymbol{A}_{\Theta}(l, l+2)=\operatorname{diag}\left[\begin{array}{lll}
1.290 & 0.086 & 0.602
\end{array}\right] \\
\boldsymbol{B}_{d 1}=\operatorname{diag}\left[\begin{array}{lll}
0.5 & 1.0 & 0.7
\end{array}\right] \\
\boldsymbol{B}_{d 2}=\operatorname{diag}\left[\begin{array}{lll}
1.0 & 0.9 & 1.1
\end{array}\right]
\end{aligned}
$$

Solving (42)-(46) applying SeDuMi package [28] in MATLAB environment, the feasible task admits

$$
\begin{aligned}
\boldsymbol{S} & =\operatorname{diag}\left[\begin{array}{lll}
2.2229 & 0.7287 & 0.8458
\end{array}\right] \\
\boldsymbol{Q} & =\operatorname{diag}\left[\begin{array}{lll}
0.1624 & 1.1587 & 0.8629
\end{array}\right] \\
\boldsymbol{R}_{1} & =\operatorname{diag}\left[\begin{array}{lll}
0.0463 & 0.1657 & 0.0609
\end{array}\right] \\
\boldsymbol{R}_{2} & =\operatorname{diag}\left[\begin{array}{lll}
0.1284 & 0.1090 & 0.1220
\end{array}\right]
\end{aligned}
$$

Using the relation defined in (27) the positive control law gain, related to the above positive solution, is

$$
\boldsymbol{K}=\left[\begin{array}{lll}
0.0208 & 0.2274 & 0.0720 \\
0.0578 & 0.1495 & 0.1443
\end{array}\right]
$$

and it is trivial to construct the underlying matrices

$$
\begin{aligned}
\boldsymbol{A}_{c} & =\left[\begin{array}{rrr}
-0.1542 & 0.7688 & 1.1097 \\
0.0132 & -3.6300 & 0.0561 \\
0.2659 & 0.2784 & -2.6171
\end{array}\right] \\
\boldsymbol{A}_{c}^{\circ} & =\left[\begin{array}{rrr}
-0.3662 & 2.3688 & 2.3097 \\
0.0232 & -6.6700 & 0.2801 \\
0.5459 & 0.8704 & -4.8011
\end{array}\right]
\end{aligned}
$$

which both are strictly Metzler and Hurwitz since

$$
\begin{gathered}
\rho\left(\boldsymbol{A}_{c}\right)=\left[\begin{array}{lll}
-0.0352 & -2.7211 & -3.6450
\end{array}\right] \\
\rho\left(\boldsymbol{A}_{c}^{\circ}=\left[\begin{array}{lll}
-0.0757 & -4.9811 & -6.7805
\end{array}\right]\right.
\end{gathered}
$$

as it is forced by (43).

As opposed to the previous part of this example a modification is given for which the following parameters are given

$$
\begin{aligned}
& \boldsymbol{A}_{1}=\left[\begin{array}{rrr}
-0.108 & 0.776 & 0.580 \\
0.032 & -1.584 & 0.080 \\
0.180 & 0.280 & -1.164
\end{array}\right] \\
& \boldsymbol{A}_{2}=\left[\begin{array}{rrr}
-0.104 & 0.824 & 0.620 \\
0.056 & -1.456 & 0.144 \\
0.100 & 0.312 & -1.020
\end{array}\right] \\
& \boldsymbol{A}_{s}=\left[\begin{array}{rrr}
-0.212 & 1.600 & 1.200 \\
0.088 & -3.040 & 0.224 \\
0.280 & 0.592 & -2.184
\end{array}\right]
\end{aligned}
$$

Solving the related corresponding LMIs set (49)-(52) results in the LMI matrix variables

$$
\begin{gathered}
\boldsymbol{S}=\operatorname{diag}\left[\begin{array}{lll}
2.2967 & 0.3120 & 0.5021
\end{array}\right] \\
\boldsymbol{R}_{1}=\operatorname{diag}\left[\begin{array}{lll}
0.0549 & 0.0820 & 0.0412
\end{array}\right] \\
\boldsymbol{R}_{2}=\operatorname{diag}\left[\begin{array}{lll}
0.1194 & 0.0580 & 0.0572
\end{array}\right]
\end{gathered}
$$

The given formula for the stabilizing feedback control law gain computation implies now

$$
\boldsymbol{K}=\left[\begin{array}{lll}
0.0239 & 0.2628 & 0.0820 \\
0.0520 & 0.1859 & 0.1139
\end{array}\right]
$$

and, consequently, using this positive $\boldsymbol{K}$ the closedloop system matrix is constructed as

$$
\boldsymbol{A}_{c}^{\circ}=\left[\begin{array}{rrr}
-0.3619 & 2.3147 & 2.3351 \\
0.0253 & -6.7382 & 0.2975 \\
0.5501 & 0.8055 & -4.7747
\end{array}\right]
$$


Obviously, $\boldsymbol{A}_{c}^{\circ}$ is a strictly Metzler and Hurwitz matrix since

$$
\rho\left(\boldsymbol{A}_{c}^{\circ}\right)=\left[\begin{array}{lll}
-0.0757 & -4.9811 & -6.7805
\end{array}\right]
$$

Because $\boldsymbol{A}$ and $\boldsymbol{A}_{s}$ are well norm-comparable while

$$
\|\boldsymbol{A}\|=3.5073, \quad\left\|\boldsymbol{A}_{s}\right\|=3.4883
$$

there exist $\alpha=0.87$ such that for $\boldsymbol{K}$ re-casted in terms of $\boldsymbol{A}_{c}^{\circ}$ one can obtain the hypothetically separated matrices

$$
\begin{gathered}
\boldsymbol{A}_{c}^{\alpha}=\boldsymbol{A}-\alpha \boldsymbol{B} \boldsymbol{K}=\left[\begin{array}{rrr}
-0.1416 & 0.7559 & 1.1553 \\
0.0245 & -3.6422 & 0.0975 \\
0.2797 & 0.2640 & -2.5669
\end{array}\right] \\
\boldsymbol{A}_{c s}^{\alpha}=\boldsymbol{A}_{s}-(1-\alpha) \boldsymbol{B} \boldsymbol{K} \\
=\left[\begin{array}{rrr}
-0.2203 & 1.5587 & 1.1799 \\
0.0008 & -3.0959 & 0.2000 \\
0.2704 & 0.5415 & -2.2077
\end{array}\right]
\end{gathered}
$$

which are both strictly Metzler and Hurwitz because their eigenvalue sets

$$
\begin{aligned}
\rho\left(\boldsymbol{A}_{c}^{\alpha}\right) & =\left[\begin{array}{lll}
-0.0069 & -2.6782 & -3.6656
\end{array}\right] \\
\rho\left(\boldsymbol{A}_{c s}^{\alpha}\right. & =\left[\begin{array}{lll}
-0.0559 & -2.2803 & -3.1878
\end{array}\right]
\end{aligned}
$$

are stable.

Metzler structures of $\boldsymbol{A}_{c}^{\circ}, \boldsymbol{A}_{c s}^{\alpha}, \boldsymbol{A}_{c s}^{\alpha}$ and their eigenvalues confirm in terms of the presented interpretations the positivity and stability of the system under control, which is the common design goal for the underlying system matrix parameters.

Eliminating the special case of purely Metzler matrices that must be treated specifically, only strictly Metzler ones are presented in Section 5, but the authors are convinced that already this explicit example helps interesting readers in understanding such defined design task in the whole complexity to bear on the presented problem.

\section{Concluding remarks}

The side of algorithmic feasibility is treated through the paper, focusing on the control law parameters in control of linear strictly Metzler time-delay systems. To indicate the routes for LMI-based algorithms, it can be underlined a common stability condition, eliminating subsystem approaches in the synthesis. The proposed methodology discuss the stability and positivity functionality of this system class in the control and it is imperative that system matrix properties are ascribed into the design condition, whilst LMI representations depend on the Metzler matrix bounds.

Moreover, since the goal was to find conditions on state feedbacks in order to get positive system gains, preserving the closed-loop system positivity, a suitable stabilizing state feedback controller design task is also presented. For obtaining the solution with desired properties of the closed-loop Metzler system, and in the feedback control design specification,
LMIs are preferred over linear programming when defining parametric boundaries. As an interest point with a general relevance within the positive system time-delay control is remarked that only not delayed system state has to be measurable. The state control problem for purely Metzler system matrices of not delayed part of the system description can be treated in a similar way using structured LMI variables and is not considered explicitly in the paper. Illustrating via examples the necessary parameter forms for the underlying LMIs structure are adduced.

The approach turns out that the realization problem may be seen as concerning a large class of positive time-delay problems. A further direction of the ongoing research is based on the study of the norm bounded Metzler systems in order to relate the framework directly to the investigation. The method needs to be expanded to adapt the proposed design way for descriptor Metzler time-delay systems in the future activity.

\section{References:}

[1] W.M. Haddad and V. Chellaboina, Stability theory for nonnegative and compartmental dynamical systems with time delay, Systems \& Control Letters, Vol. 51, No. 5, 2004, pp. 355-361.

[2] O. Mason, Diagonal Riccati stability and positive time-delay systems, Systems \& Control Letters, Vol. 61, No. 1, 2012, pp. 6-10.

[3] A.Y. Aleksandrov and O. Mason, On diagonal stability of positive systems with switches and delays Automation and Remote Control, Vol. 79, No. 12, 2018, pp. 2114-2127.

[4] L. Farina and S. Rinaldi, Positive Linear Systems. Theory and Applications, John Wiley \& Sons, New York, 2000.

[5] B.D.O. Anderson, Positive system realizations. Open Problems in Mathematical Systems and Control Theory, Springer,London, 1999, pp.7-9.

[6] A. Berman, M. Neumann, and R. Stern, Nonnegative Matrices in Dynamic Systems, John Wiley \& Sons, New York, 1989.

[7] H. Zhang, Y. Shi, J. Wang, and H. Chen, A new delay-compensation scheme for networked control systems in controller area networks, IEEE Trans. Industrial Electronics, Vol. 65, No. 9, 2018, pp. 7239-7247.

[8] L. Lu, Network controller design and analysis based on fuzzy control theory, Int. J. Circuits, Systems and Signal Processing, Vol. 13, 2019, pp. 358-365.

[9] H. Benítez-Pérez, J.L. Ortega-Arjona, P.E. Méndez-Monroy, E. Rubio-Acosta, and O.A. Esquivel-Flores, Control Strategies and 
Co-Design of Networked Control Systems. Considering Time Delay Effects, Springer Nature, Cham, 2019.

[10] M.M. Peet, A. Papachristodoulou, and S. Lall, Positive forms and stability of linear time-delay system, SIAM J. Control and Optimization, Vol. 47, No. 6, 2009, pp. 3237-3258.

[11] A. Domoshnitsky and R. Shklyar, A Positivity for non-Metzler systems and its applications to stability of time-varying delay systems, Systems \& Control Letters, Vol. 118, 2018, pp. 44-51.

[12] B. Du, On dynamic output feedback $\mathrm{H}_{\infty}$ control for positive discrete-time delay systems, Proc. 16th Int. Conf. Informatics in Control, Automation and Robotics, Vol. 1 ICINCO 2019, Prag, Czech Republic, 2019, pp. 760-766.

[13] X. Wang and J. Wen, Finite-time stabilization of switched positive systems with time-varying delays and actuator saturation Journal of Physics. Conference Series, Vol. 1848, 2021, pp. 1-7.

[14] M. Ait Rami, U. Helmke, and F. Tadeo, Positive observation problem for linear time-delay positive systems, Proc. 15th Mediterranean Conf. Control \& Automation MED'2007, Athens, Greece, 2007, pp. 1-6.

[15] R.J. Vanderbei, Linear Programming. Foundations and Extensions. Springer Nature, Cham, 2020.

[16] X. Liu, Constrained control of positive systems with delays, IEEE Trans. Automatic Control, Vol. 54, No. 7, 2009, pp. 1596-1600.

[17] G. Liu, P. Zhao, and R. Li, Stabilization of positive coupled differential-difference equations with unbounded time-varying delays, Optimal Control Applications and Methods, Vol. 2020, 2020, pp. 1-15.

[18] D. Krokavec and A. Filasová, LMI based principles in strictly Metzlerian systems control design, Mathematical Problems in Engineering, Vol. 2018, 2018, pp. 1-14.

[19] M. Akin and T. Acarman, The $\mathrm{H}_{\infty}$ model following control. An LMI approach, WSEAS Trans. Systems and Control, Vol. 15, 2020, pp. 11-18.

[20] W. Elloumi, A. Benzaouia, and M. Chaabane, Delay-dependent stabilization conditions of controlled positive continuous-time systems, Int. J. Automation and Computing, Vol. 11, No. 6, 2014, pp. 653-660.

[21] C. Briat, Stability and performance analysis of linear positive systems with delays using input-output methods, International Journal of Control, Vol. 91, No. 7, 2018, pp. 1669-1692.
[22] Y. Ebihara, Stability analysis of neutral type time-delay positive systems with commensurate delays, IFAC PapersOnLine, Vol. 50, No. 1, 2017, pp. 3093-3098.

[23] E. Fridman, Introduction to Time-Delay Systems. Analysis and Control, Springer Nature, Cham, 2014.

[24] R.A. Horn and C.R. Johnson, Matrix Analysis, Cambridge University Press, New York, 1985.

[25] D. Krokavec and A. Filasová, Mixed $\mathrm{H}_{2} / \mathrm{H}_{\infty}$ control synthesis for discrete-time linear positive systems using enhanced set of linear matrix inequalities, WSEAS Trans. Systems and Control, Vol. 15, 2020, pp. 270-281.

[26] D. Krokavec and A. Filasová, Control design for linear strictly Metzlerian descriptor systems, Proc. 18th EUCA European Control Conference ECC'20, Saint Petersburg, Russia, 2020, pp. 2092-2097.

[27] D. Krokavec and A. Filasová, $\mathrm{H}_{\infty}$ norm principle in residual filter design for discrete-time linear positive systems, European Journal of Control, Vol. 45, 2019, pp. 17-29.

[28] D. Peaucelle, D. Henrion, Y. Labit, and K. Taitz, User's Guide for SeDuMi Interface, LAASCNRS, Toulouse, 2002.

\section{Contribution of individual authors}

Anna Filasová elaborated the principles of matrix constraints representation in the control law parameter synthesis and implemented their linear matrix structures, Dušan Krokavec addressed the incidence of diagonal stabilisation principle into set of linear matrix inequalities for stability relations in control design for strictly Metzler time-delay continuous-time linear systems. Both authors have read and agreed to the proposed version of the manuscript.

\section{Acknowledgement}

The work presented in this paper was supported by VEGA, the Grant Agency of the Ministry of Education and Academy of Science of Slovak Republic, under Grant No. 1/0483/21. This support is very gratefully acknowledged.

\section{Creative Commons Attribution License 4.0 (Attribution 4.0 International, CC BY 4.0)}

This article is published under the terms of the Creative Commons Attribution License 4.0

https://creativecommons.org/licenses/by/4.0/deed.en US 